\title{
Neuronavigation and epilepsy surgery
}

\author{
Martin B. Glaser ${ }^{1 *}$, Konrad J. Werhahn ${ }^{2}$, Peter Grunert ${ }^{1}$, Clemens Sommer $^{3}$, \\ Wibke Müller-Forell ${ }^{4}$, Joachim Oertel ${ }^{1}$ \\ ${ }^{1}$ Department of Neurosurgery, University Hospital Medical Center, Johannes Gutenberg University, Mainz, Germany;
*Corresponding Author: glaserm@uni-mainz.de
${ }^{2}$ Department of Neurology, University Hospital Medical Center, Johannes Gutenberg University, Mainz, Germany
${ }^{3}$ Department of Neuropathology, University Hospital Medical Center, Johannes Gutenberg University, Mainz, Germany
${ }^{4}$ Institution of Neuroradiology, University Hospital Medical Center, Johannes Gutenberg University, Mainz, Germany
}

Received 16 February 2010; revised 11 March 2010; accepted 12 March 2010.

\begin{abstract}
Resective epilepsy surgery is an elective therapy indicated in focal epilepsy patients who are resistant to pharmacotherapy. Every effort should be undertaken to perform the procedures as safe and less traumatic as possible. Neuronavigation could represent a suitable tool to reduce surgical morbidity and increase surgical radicality. Here, we present a series of 41 patients who were operated on for medically intractable epilepsy using neuronavigation. Overall, complication rate was $17 \%$ with a favourable seizure outcome of $\mathbf{8 8 \%}$ (Engel's class I/II). Our data suggest that neuronavigation is a valuable surgical technique to accomplish a favourable outcome in epilepsy surgery.
\end{abstract}

Keywords: Neuronavigation; Epilepsy Surgery; Outcome

\section{INTRODUCTION}

Epilepsy is a frequent condition. Approximately 40 million people are affected worldwide and the prevalence of epilepsy has been estimated to be around $0.7 \%$ [1]. The mean annual incidence of first unprovoked seizures in population-based studies is 56.8 per 100000 personyears, 23.5 per 100000 person-years for single unprovoked seizures, and 33.3 per 100000 person-years for epilepsy (recurrent unprovoked seizures). Partial seizures occur in $40-60 \%$, two-thirds of which are temporal lobe epilepsies [2,3]. Clinically, focal epilepsy may first be suspected with a first witnessed report of a generalized tonic-clonic seizure but often seizures may be more subtle consisting of a transient short lasting loss of consciousness with or without oral or manual automatisms or focal tonic or clonic movements affecting parts of the body. Seizures may lead to developmental retardation, social impairment (e.g. limited choice of profession, ability to obtain a driving licence) and even sudden unexpected death in epilepsy [4]. In most cases, conservative treatment with antiepileptic drugs is successful in preventing clinical seizures, but up to 33\% of patients will prove to be resistant to medical treatment [5].

Patients with focal epilepsy are generally surgical candidates, if medical treatment with at least two different anticonvulsive drugs in sufficient doses fails and disabling seizures persist. Bad prognostic factors for medical treatment in focal epilepsy are a structural lesion on Magnetic Resonance Imaging (MRI), particularly with dual pathology, post-stroke scars and vascular malformations having the best and cortical dysgenesis and hippocampal sclerosis the poorest outcome [3]. Optimal surgical results are obtained in patients with a circumscribed seizure onset (especially temporal/temporomesial) in video-EEG recordings, concordant focal pathology on MRI (e.g. hippocampal sclerosis) and concordant neuropsychological findings [6,7].

The need for a device enabling precise introduction of instruments into deep intracerebral structures was first addressed by Zernov et al. [8] 1890. He constructed a frame which was fixed on the skull by screws. The position of deep structures were measured from external anatomical landmarks. Clark developed 1908 a frame which served as a stable coordinate system for calculation of intracranial targets in relation to the frame $[9,10]$. In the second half of the last century these frames were refined. More and more indications were found along with the progress of the imaging modalities (x-ray, angiogram, computed tomography, MRI). Frame based stereotactic systems are still the most accurate navigational tools and very small targets like the subthalamic nucleus can be implanted with depth electrodes for the treatment of parkinsonism. 
One major disadvantage of the stereotactic frame is the restricted surgical field as long as the arc is in place. At the end of the 1980s the "frameless" navigation was developed, with first clinical applications in neurosurgery at the beginning of the 1990s [11].

Nowadays, frameless neuronavigation is an accepted tool in contemporary microneurosurgery [12-15]. Its application contributes to make surgical approaches smaller and less invasive [16]. Consequently neuronavigation was integrated also in epilepsy surgery [17].

The neuronavigation is basically a miniature of a GPS (general positioning system). The neuronavigation systems are able to determine the position of the tip of a pointer in 3-D-space and to transfer the position into the appropriate CT or MRI data set in real time during the entire operation (in case of a microscope the focus corresponds to the tip of the pointer). From the technical point of view we can distinguish between armbased and armless navigation. The latter have the advantage not to restrict the operative field. Different armless systems were realized using sonic, infrared, magnetic waves or visible light (see Figure 1). The transfer of the pointer tip in the appropriate images makes a registration before application necessary. Per point registration and surface registration were developed for this purpose. The navigation devices have higher flexibility but less accuracy in comparison to the frame based systems. Regarding navigation accuracy we have clearly to distinguish between technical accuracy of the navigation system (how accurately the system determines the position in the 3-Dspace), registration accuracy (how accurately is the data transfer from 3-D-space into the CT and MRI image space) and application accuracy depending of the intraoperative situation including brain shift [18].

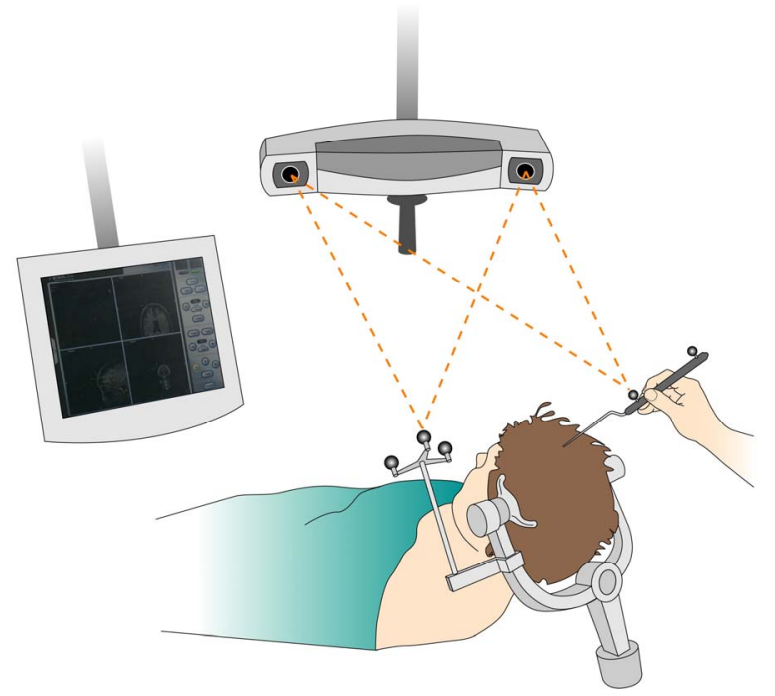

Figure 1. Drawing of an armless neuronavigation system setup.
For this study, we reviewed our surgical cases that were performed for pharmacoresistent focal epilepsy using a neuronavigation device.

\section{MATERIALS AND METHODS}

In our retrospective study, we gathered the clinical data of all patients who had navigation assisted surgery for medically intractable epilepsy. We evaluated the charts of 41 patients who were treated in our institution from 09.2003 to 08.2009 and reviewed the postoperative clinical follow up as well as neuro-imaging data for the degree of resection and complications.

Initially we used the Optical Tracking System (OTS®, Radionics, Burlington, Massachusetts, USA). In 31 cases, we navigated with the BrainLAB ${ }^{\circledR}$ System (BrainLAB, Heimstetten, Germany) and in a further 9 cases with the SonoWand ${ }^{\circledR}$ (Mison, Trondheim, Norway).

In frameless Neuronavigation, after general anaesthesia has been induced and immediately before surgery the patient's head is fixed in a three point fixation device and then referenced to the presurgical MRI (or other imaging modality such as computed tomography) by indicating to at least 4 defined landmarks so that the navigation system may locate the patient's head in the three dimensional space. Hereafter the patient's individual anatomy is shown on a monitor according to the region where a pointer is held on. The surgeon sees exactly where the targeted lesion is in relation to the skull surface to place the craniotomy on the ideal site. Moreover, he may check the position of his instrument any time during surgery.

For selective amygdala-hippocampectomies, we used a supraorbital craniotomy via a subfrontal approach [19]. Temporal pole resections with amygdala-hippocampectomies were approached via a small anterior temporal craniotomy (diameter approx. $2.5 \mathrm{~cm}$ ). For extratemporal lesionectomies neuronavigation was also employed to gain direct access with craniotomies as small as possible. "Keyhole" approaches were applied when possible, especially in deeper seated lesions.

\section{PATIENTS}

This series includes 41 consecutive patients with pharmacoresistent focal epilepsy with a mean age of 36 years (15-70 years). There were 17 male and 24 female individuals. The mean duration of the epilepsy was 15.8 years. Most patients suffered from mesial temporal lobe epilepsy ( $n=28,17$ left/11 right). All of them had been transferred from the department of neurology of the University Medical Center, Mainz, after video-EEGmonitoring for identification of the seizure onset region, 
correlation with the neuro-imaging and neuropsychological testing. Histological findings showed hippocampal sclerosis in 21 specimens. The remaining 7 had no specific changes (no abnormality, dysplasias, corpora amylacea).

The extra-temporomesial pathologies consisted of 4 gangliogliomas, 1 gangliocytoma, 2 astrocytomas, 1 oligoastrocytoma, 2 cavernomas, 1 gliosis after hemorrhage from an AVM, 1 dermoid and 1 meningeoma (6 left/7 right).

\section{SURGICAL PROCEDURES}

The surgery for the mesial temporal lobe epilepsy patients consisted of 2 selective amygdalahippocampectomies via a supraorbital subfrontal approach. The remaining 27 cases had an anterior temporal craniotomy for pole resection and amygdalahippocampectomy.

The extratemporal pathologies were approached by the shortest or least traumatic way concerning the patient's neurological function. On the BrainLAB planning station, it is possible to determine the trajectory and import the information of the presurgical MRIs into the intraoperative surgical field.

In the operating room neuronavigation was installed after fixation of the patient's head in the Mayfield clamp. Accuracy was checked by correlation with anatomical

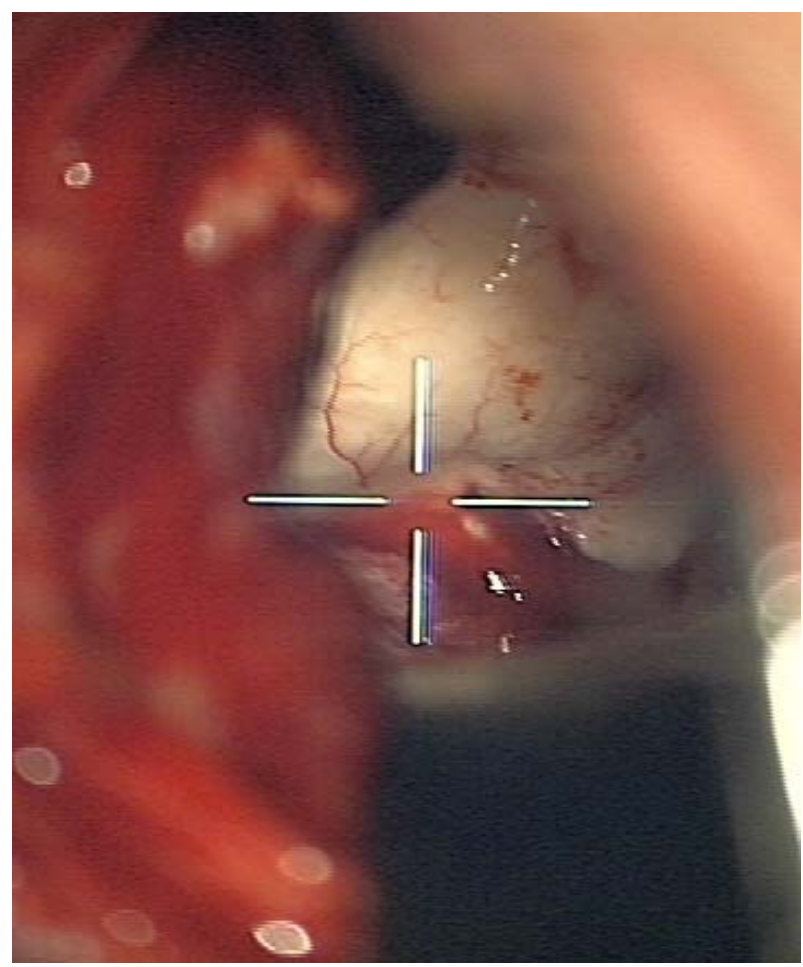

Figure 2. View of the hippocampus through the navigated microscope. landmarks after referencing the patients head with the preoperative 3-D-MRI data set either by laser or landmark registration (at least 4 points; mostly nasion, lateral orbital rims and upper helix attachments).

Neuronavigation was used to gain direct access to the pathological structures. This was achieved generally by use of a pointer. Additionally the microscope (Pentero or NC4, Carl Zeiss, Oberkochen, Germany) itself could be registered and navigated with the BrainLAB system. It was especially helpful in the amygdala-hippocampectomies in opening the temporal horn of the lateral ventricle to enable the dissection of the hippocampus. The viewing direction could be brought in the planned trajectory to reach the targeted structure. When the target is displayed in the ocular of the microscope, it is not necessary for the surgeon to place a pointer in the surgical field and look up to the monitor of the navigation system.

Finally the neuronavigation was then used to "define" the extent of resection of the hippocampus. It was intended to remove it at least to the dorsal edge of the cerebral peduncle.

\section{DATA EVALUATION/FOLLOW UP}

For all patients, site of surgery, duration between completed anaesthesiological preparation and skin incision as well as the time for the surgery itself, blood loss, ICU stay, hospital stay, neurological detoriation after surgery, degree of resection and seizure outcome were collected.

The follow up of the patients and the classification concerning Engel's epileptological outcome classes [20] were provided by the referring neurological department (KJW). Mean follow up time was 23 month.

\section{RESULTS}

Installation and usage of the neuronavigation systems was possible in all procedures. Average patient preparation (positioning, head fixation, referencing the neuronavigation, shaving, skin prepping, sterile draping) took 37 minutes. Mean duration of surgery was 209 minutes from skin incision to wound closure. The mean ICU stay scored 20.3 hours, the mean hospital stay 8.5 days. There was an average blood loss of 310 cc per complete procedure. Not a single blood product was administered.

There was no mortality in this series. The following complications were noted: One patient had a space occupying frontal epidural haematoma on his routine postoperative cranial computed tomogram which was clinically asymptomatic but evacuated for its size. Two patients showed a slight hemiparesis caused by small thalamic ischemias. They regained full strength but still 
have a deficit in fine motor skills. A further two patients had incomplete oculomotor palsies which resolved without sequelae. One patient developed a severe generalized vasospasm 10 days after subtotal frontal lobectomy. He has no focal neurological deficit but a relevant lack of motivation.

One rhinoliquorrhoea occurred after a supraorbital approach via the opened frontal sinus. The liquorrhoea ceased after temporary lumbar drainage.

Postsurgical imaging showed complete removal of the extratemporal pathologies in 9 of the 13 cases. The degree of hippocampal resection was noted in relation to the brain stem: a relatively short resection of the hippocampus only to the middle of the cerebral peduncle was performed in 5 cases, to the dorsal margin of the cerebral peduncle in 20 cases and in a further 3 cases beyond.

The neuronavigation was sufficiently exact in all cases at the beginning of the procedure. Accuracy was as reliable with laser patient registration as with registration via anatomical landmarks. The calculated mean deviation was $1.7 \mathrm{~mm}$. It was possible to reach all lesions/ structures that were aimed for. It was extremely helpful in localization of the temporal horn in amygdala-hippocampectomies. Neuronavigation overestimated the degree of resection of the hippocampus, possibly due to brain shift after CSF loss-especially after opening of the lateral ventricle.

Postoperative seizure outcome was favourable after amygdala-hippocampectomy with 21 patients Engel's class I and 6 patients Engel's class II. One patient was seizure free for 3.5 years and developed pharmacoresistent temporal lobe epilepsy again so that re-resection is being considered.

In the patients group with the extratemporal resections, 11 patients became seizure free (Engel's class I). Two patients did not profit at all and have still the same seizure frequency in comparison to the presurgical state (partial tumor resections).

In total, antiepileptic drugs were discontinued in 8 patients and reduced in 5. The majority of 29 patients is still under medication, similar to presurgical status.

\section{DISCUSSION}

For decades, atraumatic surgery for medically refractory epilepsy has been the objective in order to improve patients functions and at the same time effectively reduce seizures. Neuronavigation contributes to that aim by minimizing the craniotomies and reach the target in the planned trajectory [13].

On the other hand, there are only few publications concerning neuronavigation and resective epilepsy surgery [17,21-25].

Previous reports on neuronavigation in epilepsy surgery were published without discussing its advantages and pitfalls or without giving any clinical data [26-28].

Wurm et al. [24] published the largest series of 140 patients who underwent surgery for medically intractable epilepsy. After the procedure for miscellaneous pathologies surgeons answered a questionnaire to assess the impact of the neuronavigation. They concluded that the application of the navigation system was effectively and safe in terms that the targets, even small in size, could be located precisely and electrodes could be placed accurately as well. Moreover the approach could be individually tailored.

In a previous series of Oertel et al. [22] neuronavigation seemed to be helpful in avoidance of complications ( $8 \%$ vs. $22 \%$ ). In $93 \%$ the surgeon rated the application of the neuronavigation as "helpful".

A comparison of the complications in various studies is compiled in Table 2, seizure outcome in Table 3.

In our series complication rate and seizure outcome are comparable to larger series [29]. The application was safe. There were no complications with direct referral to the use of the navigation system. The time for preparation of the navigation was acceptable: in our evaluation the total time from anaesthesia induction to skin incision was 37 minutes. In comparison to that the installation of the neuronavigation equipment alone took additional 26 minutes in another study [30]. Surgery itself was not prolonged.

Table 1. Usefulness of neuronavigation.

\begin{tabular}{ll}
\hline $\begin{array}{l}\text { Presurgical planning/strategy } \\
\text { Determination of craniotomy site }\end{array}$ & Helpful for studying patients individual anatomy \\
Locating lesions & Helpful, especially in subcortical pathologies \\
Amygdala-hippocampectomies & Extreme helpful in access the temporal horn \\
Resection control & Variable (brain shift), often overestimation, consider alternatives (e.g. ultrasound) \\
Delicate site of surgery & Helpful, shows eloquent structures as well
\end{tabular}


Table 2. Epilepsy surgery and complications (perm. = permanent; trans. = transient).

\begin{tabular}{|c|c|c|c|c|c|}
\hline Complications & Acar et al. & Oertel et al. & Cho et al. & Glaser et al. & Sindou et al. without Navigation \\
\hline CSF fistula & & & 2 trans. & 1 trans. & \\
\hline Visual field defects & 4 perm. & Not investigated & 1 & 4 perm. & Not investigated \\
\hline CN palsy & & 1 trans. & & 2 trans. & \\
\hline Motor deficit & 1 perm. & 1 trans. & & 2 trans. & 2 perm. \\
\hline Aphasia & 1 trans. & 1 perm. & & 1 trans. & \\
\hline Postop. haematoma & & & 1 & 1 & 3 \\
\hline \multirow[t]{2}{*}{ Infection } & & & & & 3 \\
\hline & $\mathrm{n}=39$ & $\mathrm{n}=38$ & $\mathrm{n}=46$ & $\mathrm{n}=41$ & $\mathrm{n}=100$ \\
\hline
\end{tabular}

Table 3. Seizure-outcome after epilepsy surgery.

\begin{tabular}{|c|c|c|c|c|c|}
\hline Engel's class & Acar et al. & Oertel et al. & Cho et al. & Glaser et al. & Sindou et al. without Navigation \\
\hline I & 37 (95\%) & $20(53 \%)$ & $28(61 \%)$ & $32(78 \%)$ & 85 (85\%) \\
\hline II & $2(5 \%)$ & & $10(22 \%)$ & $4(10 \%)$ & $9(9 \%)$ \\
\hline III & & & $6(13 \%)$ & $2(5 \%)$ & $2(2 \%)$ \\
\hline \multirow[t]{2}{*}{ IV } & & & $2(4 \%)$ & $3(7 \%)$ & $4(4 \%)$ \\
\hline & $\mathrm{n}=39$ & $\mathrm{n}=38$ & $\mathrm{n}=46$ & $\mathrm{n}=41$ & $\mathrm{n}=100$ \\
\hline
\end{tabular}

\section{CONCLUSIONS}

Based on these results and our experience in the use of neuronavigation, we conclude that the application of a navigation system in epilepsy cases is safe and helpful in finding the targeted structure and in minimizing trauma to the patient by smaller craniotomies.

\section{REFERENCES}

[1] Hauser, W.A., Annegers, J.F. and Kurland, L.T. (1991) Prevalence of epilepsy in Rochester, Minnesota: 19401980. Epilepsia, 32(4), 429-445.

[2] Olafsson, E., Ludvigsson, P., Gudmundsson, G., Hesdorffer, D., Kjartansson, O. and Hauser, W.A. (2005) Incidence of unprovoked seizures and epilepsy in Iceland and assessment of the epilepsy syndrome classification: A prospective study. The Lancet Neurology, 4(10), 627634.

[3] Semah, F., Picot, M.C., Adam, C., Broglin, D., Arzimanoglou, A., Bazin, B., Cavalcanti, D. and Baulac, M. (1998) Is the underlying cause of epilepsy a major prognostic factor for recurrence? Neurology, 51(5), 12561262.

[4] Kloster, R. and Engelskjon, T. (1999) Sudden unexpected death in epilepsy (SUDEP): A clinical perspective and a search for risk factors. Journal of Neurology, Neurosur- gery \& Psychiatry, 67(4), 439-444.

[5] Sillanpaa, M. and Schmidt, D. (2006) Natural history of treated childhood-onset epilepsy: Prospective, long-term population-based study. Brain, 129(Pt3), 617-624.

[6] Tonini, C., Beghi, E., Berg, A.T., Bogliun, G., Giordano, L., Newton, R.W., Tetto, A., Vitelli, E., Vitezic, D. and Wiebe, S. (2004) Predictors of epilepsy surgery outcome: A meta-analysis. Epilepsy Research, 62(1), 75-87.

[7] Berg, A.T., Vickrey, B.G., Langfitt, J.T., Sperling, M.R., Walczak, T.S., Shinnar, S., Bazil, C.W., Pacia, S.V. and Spencer, S.S. (2003) The multicenter study of epilepsy surgery: Recruitment and selection for surgery. Epilepsia, 44(11), 1425-1433.

[8] Zernov, D. (1890) L’encephalometre. revue générale de clinique et de thérapeutique, 19, 320.

[9] Horsley, V.R.C. (1908) The structure and functions of the cerebellum examined by a new method. Brain, 31(1), 45-124.

[10] Kirschner, M. (1933) Die Punktionstechnik und die Elektrokoagulation des Ganglion Gasseri. Über gezielte Operationen. Langenbecks Archiv für klinische Chirurgie, 176, 581-620.

[11] Grunert, P., Darabi, K., Espinosa, J. and Filippi, R. (2003) Computer-aided navigation in neurosurgery. Neurosurgical Review, 26(2), 73-99.

[12] Enchev, Y. (2009) Neuronavigation: Geneology, reality, and prospects. Neurosurgical Focus, 27(3), E11.

[13] Ganslandt, O., Behari, S., Gralla, J., Fahlbusch, R. and Nimsky, C. (2002) Neuronavigation: Concept, techniques 
and applications. Neurol India, 50(3), 244-255.

[14] Wagner, W., Gaab, M.R., Schroeder, H.W. and Tschiltschke, W. (2000) Cranial neuronavigation in neurosurgery: Assessment of usefulness in relation to type and site of pathology in 284 patients. Minim Invasive Neurosurg 43(3), 124-131.

[15] Spetzger, U., Laborde, G. and Gilsbach, J.M. (1995) Frameless neuronavigation in modern neurosurgery. Minim Invasive Neurosurg, 38(4), 163-166.

[16] Winkler, D., Lindner, D., Strauss, G., Richter, A., Schober, R. and Meixensberger, J. (2006) Surgery of cavernous malformations with and without navigational support-a comparative study. Minimally Invasive Neurosurgery, 49(1), 15-19.

[17] Stone, S.S. and Rutka, J.T. (2008) Utility of neuronavigation and neuromonitoring in epilepsy surgery. Neurosurgical Focus, 25(3), E17.

[18] Nimsky, C., Ganslandt, O., Hastreiter, P., Wang, R., Benner, T., Sorensen, A.G. and Fahlbusch, R. (2005) Preoperative and intraoperative diffusion tensor imaging- based fiber tracking in glioma surgery. Neurosurgery 56(1), 130-137.

[19] Reisch, R., Stadie, A., Kockro, R., Gawish, I., Schwandt, E. and Hopf, N. (2009) The minimally invasive supraorbital subfrontal key-hole approach for surgical treatment of temporomesial lesions of the dominant hemisphere. Minim Invasive Neurosurg, 52(4), 163-169.

[20] Engel, J.V.N.P. Jr., Rasmussen, T.B. and Ojemann, L.M. (Ed.), (1993) Outcome with respect to epileptic seizures. Raven Press, New York.

[21] Miyagi, Y., Shima, F., Ishido, K., Araki, T., Taniwaki, Y., Okamoto, I. and Kamikaseda, K. (2003) Inferior temporal sulcus approach for amygdalohippocampectomy guided by a laser beam of stereotactic navigator. Neurosurgery 52(5), 1117-1123.

[22] Oertel, J., Gaab, M.R., Runge, U., Schroeder, H.W., Wagner, W. and Piek, J. (2004) Neuronavigation and complication rate in epilepsy surgery. Neurosurgical Review, 27(3), 214-217.

[23] Wurm, G., Wies, W., Schnizer, M., Trenkler, J. and Holl, K. (2000) Advanced surgical approach for selective amygdalohippocampectomy through neuronavigation. Neurosurgery, 46(2), 1377-1382.

[24] Wurm, G., Ringler, H., Knogler, F., Schnizer, M. (2003) Evaluation of neuronavigation in lesional and non-lesional epilepsy surgery. Computer Aided Surgery, 8(4), 204-214.

[25] Hirabayashi, H., Chitoku, S., Hoshida, T. and Sakaki, T. (1999) Accuracy and availability of the computed assisted neurosurgery navigation system during epilepsy surgery. Stereotact Funct Neurosurg, 72(2-4), 117-124.

[26] Acar, G., Acar, F., Miller, J., Spencer, D.C. and Burchiel, K.J. (2008) Seizure outcome following transcortical selective amygdalohippocampectomy in mesial temporal lobe epilepsy. Stereotact Funct Neurosurg, 86(5), 314319.

[27] Fahlbusch, R., Ganslandt, O. and Nimsky, C. (2000) Intraoperative imaging with open magnetic resonance imaging and neuronavigation. Child's Nervous System, 16(10-11), 829-831.

[28] Wheatley, B.M. (2008) Selective amygdalohippocampectomy: The trans-middle temporal gyrus approach. Neurosurg Focus, 25(3), E4.

[29] Sindou, M.G.M., Isnard, J., Ryvlin, P., Fischer, C. and Mauguière, F. (2006) Temporo-mesial epilepsy surgery: outcome and complications in 100 consecutive adult patients. Acta Neurochir (Wien), 148(1), 39-45

[30] Willems, P.W.A., Taphoorn, M.J.B., Burger, H., van der Sprenkel, J.W.B. and Tulleken, C.A.F. (2006) Effectiveness of neuronavigation in resecting solitary intracerebral contrast-enhancing tumors: A randomized controlled trial. Journal of Neurosurgery, 104(3), 360-368. 